\title{
FATORES QUE INTERFEREM NA ADESÃO ȦS PRECAUÇÕES PADRÃO POR PROFISSIONAIS DA SAÚDE: REVISÃO INTEGRATIVA
}

\section{Quézia Boeira da Cunha1, Silviamar Camponogara², Etiane de Oliveira Freitas ${ }^{3}$, Camila Pinno $^{3}$, Gisele Loise Dias $^{3}$,} Mariana Pellegrini Cesar ${ }^{4}$

Objetivo: identificar os fatores que interferem na adesão às precauções padrão por trabalhadores de saúde em instituições hospitalares. Metodologia: revisão integrativa conduzida nas bases de dados Literatura Latino-Americana e do Caribe em Ciências da Saúde, US National Library of Medicine e SciVerse Scopus. Resultados: dezesseis estudos atenderam aos critérios estabelecidos. Ficaram evidentes fatores que influenciam na adesão: fatores individuais - conscientização, percepção do risco, percepção da eficácia das medidas de proteção, crenças e valores, conhecimento, subjetividade, relações interpessoais; fatores relacionados ao trabalho - carga de trabalho, obstáculos do trabalho; e fatores organizacionais - disponibilidade de equipamento de proteção individual, estrutura física, supervisão, ações gerenciais. Conclusão: vários fatores interferem na adesão às precauções padrão, sendo fundamental promover aqueles que irão levar à adoção universal e minimizar os que impedem sua plena utilização.

Descritores: Precauções universais, Equipamento de proteção individual, Saúde do trabalhador, Pessoal de saúde.

\section{FACTORS THAT INTERFERE WITH ADHERENCE TO STANDARD PRECAUTIONS FOR HEALTH PROFESSIONALS: INTEGRATIVE REVIEW}

Objective: To identify the factors that interferes with adherence to standard precautions for health workers in hospitals. Methodology: Integrative review conducted in the databases Literatura Latino-Americana e do Caribe em Ciências da Saúde, US National Library of Medicine e SciVerse Scopus. Results: Sixteen studies met the inclusion criteria. The factors that influence with adherence to standard precautions was evident: individual factors - awareness, risk perception, perception of the effectiveness of protective measures, beliefs and values, knowledge, subjectivity, interpersonal relationships; work factors - workload, work obstacles; and organizational factors - availability of individual protection equipment, physical structure, supervision, management actions. Conclusion: Several factors interfere in adherence to standard precautions, and it is fundamental to promote those that will lead to universal adoption and minimize those that prevent its full use.

Descriptors: Universal precautions, Personal protective equipment, Occupational health, Health personnel.

\section{FACTORES QUE INTERFEREN CON LA ADHESION DE PRECAUCIONES ESTÁNDAR POR PROFESIONALES DE SALUD: REVISIÓN INTEGRADORA}

Objetivo: Identificar los factores que interfieren en la adhesión de precauciones estándar para trabajadores de salud en los hospitales. Metodología: Revisión integradora realizada en bases de datos Literatura Latino-Americana e do Caribe em Ciências da Saúde, US National Library of Medicine e SciVerse Scopus. Resultados: Dieciséis estudios cumplieron los criterios de inclusión. Fue factores evidentes que influyen en la adhesión: factores individuales - sensibilización, percepción de riesgo, percepción de la eficacia de las medidas de protección, creencias y valores, conocimiento, subjetividad, relaciones interpersonales; factores de trabajo - carga de trabajo, obstáculos de trabajo; y factores de organización - disponibilidad de EPI, estructura física, supervisión, acciones de manejo. Conclusión: Hay varios factores que influyen en la adhesión a las precauciones estándar, siendo esencial para potenciar los que dará lugar a la adopción universal y reducir al mínimo las que impiden su plena utilización. Descriptores: Precauciones universales, Equipo de protección personal, Salud laboral, Personal de salud.

'Enfermeira. Mestre. Universidade Federal de Santa Maria - UFMS. quezinhacunha@hotmail.com³

²Enfermeira. Doutora. Docente da Universidade Federal de Santa Maria.

${ }^{3}$ Enfermeira. Universidade Federal de Santa Maria.

${ }^{4}$ Enfermeira. Centro Universitário Franciscano de Santa Maria. 


\section{INTRODUÇÃO}

Os trabalhadores da saúde são vulneráveis ao risco biológico devido à exposição que ocorre durante o manuseio direto com sangue e fluidos corpóreos dos pacientes, que são possíveis fontes de transmissão de patógenos ${ }^{(1)}$. As precauções padrão (PP) são medidas de proteção que devem ser executadas, pelos trabalhadores, considerando que quaisquer fluídos corporais (exceto suor) podem conter agentes infectantes ${ }^{(2)}$.

Muitas vezes, os trabalhadores executam suas tarefas sem o uso adequado de equipamentos de proteção individual (EPI) e sem a preocupação com a exposição aos riscos ${ }^{(3,4)}$, demonstrando a carência de uma cultura de segurança frente ao risco biológico(5). Os trabalhadores conhecem os riscos a que estão expostos, entretanto, esse conhecimento não se transforma em ações seguras de prevenção de acidentes $^{(6)}$.

No Brasil, apesar de existir uma legislação que contempla grande parte das recomendações internacionais, quanto à exposição ocupacional aos agentes biológicos, percebe-se a persistência da ideia de que o risco é inerente à atividade e que a exposição a esse risco é aceitável ${ }^{(7)}$. A prevenção dos acidentes de trabalho com fluidos biológicos, bem como o monitoramento pós-acidente, é papel fundamental das instituições de saúde para valorização dos trabalhadores ${ }^{(8)}$.

O uso adequado de medidas de proteção é um tema complexo e que pode sofrer influência de diversos fatores. Há necessidade de se discutir questões que envolvem a biossegurança no desenvolvimento das atividades laborais, com foco para a diminuição dos riscos ocupacionais ${ }^{(9)}$. Este estudo teve como objetivo identificar os fatores que interferem na adesão às PP, por trabalhadores de saúde, em instituições hospitalares.

\section{METODOLOGIA}

Revisão integrativa da literatura, método de pesquisa que percorre as seguintes etapas ${ }^{(10)}$ : identificação do tema e seleção das questões de pesquisa, estabelecimento de critérios de inclusão e exclusão, seleção dos estudos, definição das informações a serem extraídas dos estudos selecionados, avaliação das evidências e análise (categorização), discussão e apresentação da sintese do conhecimento evidenciado nos estudos analisados.

A busca desenvolveu-se nas bases de dados: Literatura Latino-Americana e do Caribe em Ciências da Saúde (LILACS), US National Library of Medicine (PUBMED) e SciVerse Scopus (SCOPUS). Na estratégia de busca utilizada na LILACS, foram utilizados os descritores: "exposição a agentes biológicos" or "exposição ocupacional" or "precauções universais" and "medidas de segurança" or "equipamentos de proteção". Na PUBMED, utilizou-se como estratégia de busca "exposure, occupational" (MESH) and "precautions, universal" (MESH) and "nursing" (all fields) and "1996-2015". Já na SCOPUS, realizou-se a pesquisa com as palavras "exposure to biological agents" (TITLE, ABSTRACT, KEYWORDS) e os filtros de tempo (a partir de 1996) e subárea (enfermagem).

O levantamento dos estudos ocorreu em fevereiro de 2016. Os critérios de inclusão foram: estudos originais que investigaram os fatores que interferem na adesão às PP entre trabalhadores da saúde em ambiente hospitalar, publicados em português, inglês ou espanhol, no período de 1996 até 2015, que estivessem disponiveis online na íntegra gratuitamente. $O$ marco temporal deve-se ao fato de que as PP terem sido instituidas no ano de 1996, pelo Center for Disease Control and Prevention (CDC). Os artigos duplicados foram considerados apenas uma vez. A Figura 1 demonstra o resultado da busca.

Figura 1 - Estrutura do desenvolvimento do estudo de revisão. LILACS, SCOPUS, PUBMED, 2016

\section{Busca eletrônica nas \\ bases de dados LILACS, PUBMED e SCOPUS}

Identificação de 145 produções

Leitura do título e resumo (113 exclusões por recorte temático)

32 produções

Busca dos artigos na íntegra (06 exclusões por não estarem disponiveis online gratuitamente)

26 produções Leitura na íntegra (10 exclusões por não responderam à questão de pesquisa)

16 produções

CORPUS DA PESOUISA

Foram encontrados 145 estudos, sendo 113 excluídos por não abordarem a temática de interesse, seis excluídos por não estarem disponiveis online na integra gratuitamente. Dos 26 artigos que foram selecionados para análise, 16 responderam à questão de pesquisa e constituíram o corpus do estudo (Figura 1). Após leitura exaustiva, os estudos primários foram submetidos à mesma extração e coleta de dados, por meio do preenchimento de um quadro tipo sinóptico. Os artigos foram identificados pela letra A de "artigo", seguida de uma numeração (Al, A2, A3, sucessivamente). Com relação às questões éticas, foram respeitados os preceitos de autoria. 


\section{RESULTADOS}

Quanto à origem dos estudos, o Brasil concentrou a maior parte das publicações (43,75\%). A população estudada contemplou trabalhadores da enfermagem (75\%) e, também, estudos com equipe de enfermagem e equipe médica conjuntamente (25\%). O cenário das pesquisas foi, majoritariamente, hospitais gerais (56,25\%), com alguns estudos realizados em setores específicos. Referente ao delineamento metodológico, houve predomínio de estudos quantitativos (68,75\%). O Quadro 1 apresenta a identificação, autores, título, ano de publicação e periódico dos estudos.

Quadro 1 - Artigos que compõem o corpus da pesquisa, por autores, título, ano, periódico e país.

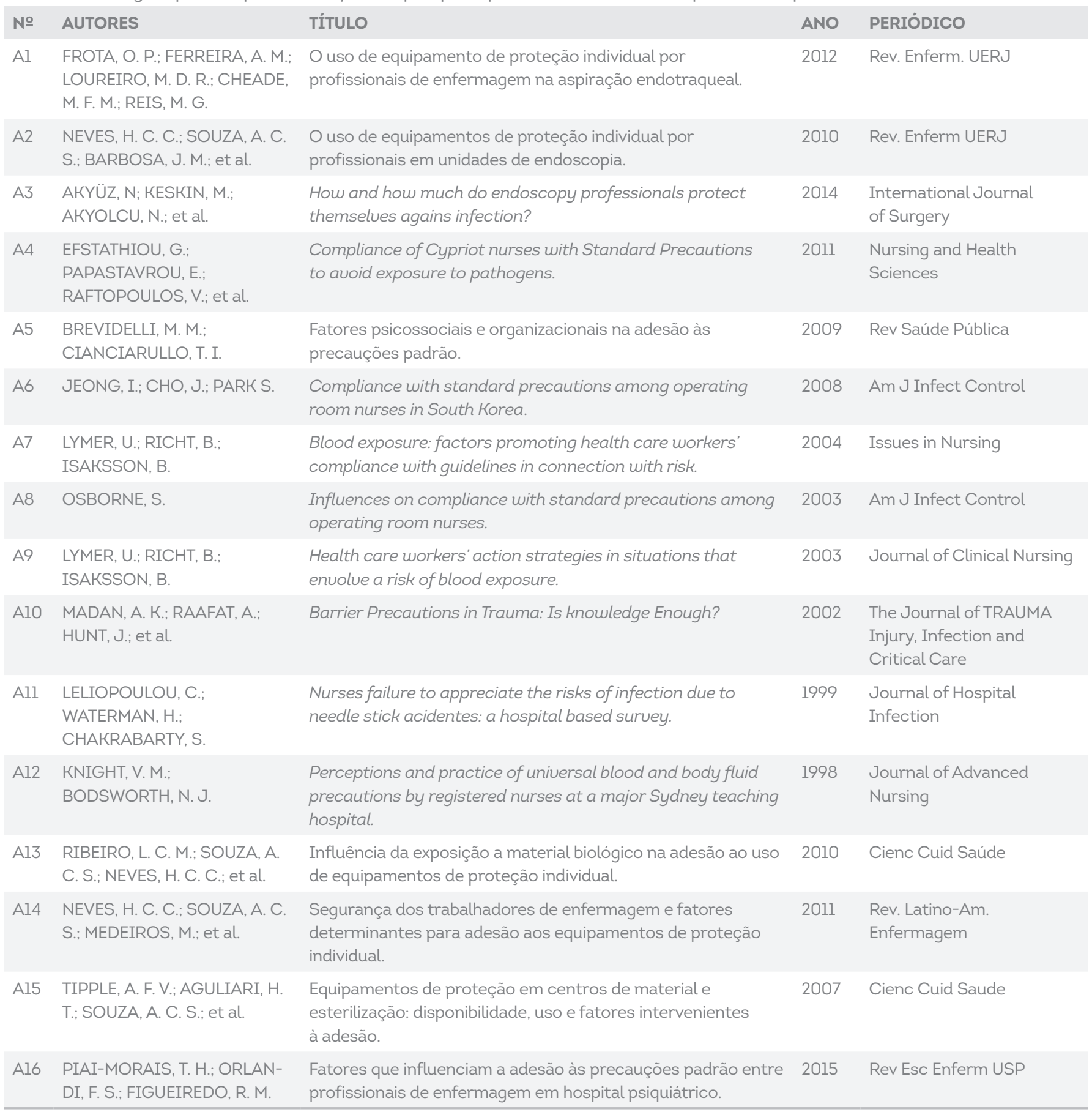

Fonte: resultados da pesquisa.

A partir da análise dos estudos, evidenciaram-se fatores que interferem na adesão dos trabalhadores da saúde às PP. Esses fatores foram agrupados em três categorias: fatores individuais, fatores relacionados ao trabalho e fatores organizacionais. Na Figura 2, são identificados os principais fatores que interferem na adesão às PP. 
Figura 2 - Apresentação esquemática dos fatores que interferem na adesão às PP.

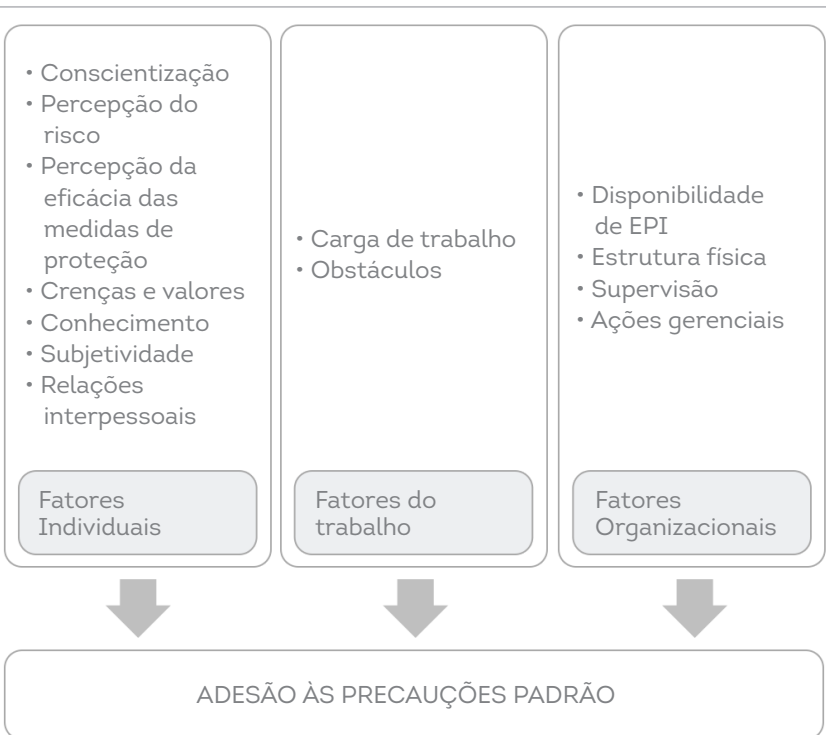

\section{DISCUSSÃO}

Dentre os fatores que interferem na adesão do profissional de saúde às PP, diversas investigações têm demonstrado a importância dos fatores individuais. A conscientização do profissional e a percepção dos riscos ocupacionais são mencionadas em diversos estudos ${ }^{(11-17)}$. Autores ${ }^{(13)}$ afirmam que a consciência de estar em risco constante influência o comportamento dos profissionais. A convivência permanente com a exposição ao material biológico favorece a diminuição da percepção dos riscos pelos profissionais, dificultando a tomada de decisão para a adoção das $\mathrm{PP}^{(16)}$

Algumas vezes, a compreensão do trabalhador quanto à necessidade de adesão às PP é maior que a adesão em si ${ }^{(17)}$. Apesar de representar proteção, as PP não livram totalmente o profissional do risco de se acidentar e adquirir uma doença ocupacional(16). A percepção de ineficiência das medidas de proteção pode influenciar as atitudes adotadas diante de situações de risco. Nesse sentido, a eficácia das medidas de proteção, percebida pelo profissional, é o que direciona a ação e o comportamento do mesmo frente ao risco(6).

Os fatores individuais talvez sejam os mais complexos, considerando que a percepção dos riscos é a primeira tarefa para o uso de $\mathrm{PP}^{(17)}$. A falta do medo de se contaminar e a crença de que nada irá acontecer reforçam o sentimento da autoconfiança do trabalhador, o que leva ao descaso no uso do EPI e propicia a (des)proteção(6,11)

A falta de conhecimento com relação ao risco e ao tipo de EPI indicado, para cada procedimento, também é um fator que leva os profissionais a não utilizarem os EPI ou a fazerem o uso inadequado dos mesmos ${ }^{(11,12,18)}$. Estudo(18) verificou associação significativa entre bons conhecimentos e o risco reduzido de exposição ocupacional, demonstrando que o conhecimento é um importante fator de promoção do uso de medidas de proteção.

As estratégias educativas associadas à avaliação, revisão e exigência de práticas seguras no processo de trabalho são apontadas como fundamentais para elevar o nivel de adesão às $\mathrm{PP}^{(19-22)}$. A educação do trabalhador, por meio da reflexão acerca de sua prática, pode constituir-se em um fator positivo para mudanças preventivas. A ocorrência de acidentes com material biológico pode ser uma oportunidade de problematizar essa experiência e promover a busca da reflexão crítica dos trabalhadores ${ }^{(19)}$.

Além de treinamento regular, é preciso incentivar a participação dos trabalhadores no desenvolvimento de diretrizes e regulamentos, pois o envolvimento voluntário nesse processo pode contribuir para aumentar a adesão(21). Nesse sentido, existe a necessidade de produção de políticas para lidar com as percepções de riscos dos trabalhadores ${ }^{(15)}$.

Por outro lado, o conhecimento nem sempre é suficiente para evitar a exposição biológica. A análise das razões, atitudes e crenças de trabalhadores de enfermagem demonstrou que, mesmo em grupos que conhecem os riscos, existe uma média suscetibilidade dos trabalhadores à exposição biológica(b) Isso evidencia que a adesão às medidas de proteção está relacionada a aspectos que vão além de ter o conhecimento sobre o risco.

A subjetividade dos trabalhadores precisa ser levada em consideração. Estudo(23) com abordagem qualitativa apontou que os profissionais vivem um conflito entre diferentes demandas. Esse estudo também revelou que os profissionais que protegem a sua própria integridade biológica podem sentirse envergonhados perante pacientes ou colegas de trabalho por parecerem mais ansiosos ${ }^{(23)}$

Quanto aos fatores relacionados ao trabalho, a carga de trabalho demonstrou forte relação com questões de segurança. Trabalhadores da enfermagem da Suécia afirmaram que o aumento da carga de trabalho teve um impacto negativo nas condições de segurança(23). Em outro estudo, o "tempo" foi apontado, por $61 \%$ dos participantes, como um fator que não interfere na adesão às PP(22). Além disso, o dilema gerado entre salvar a vida do paciente e cuidar da própria proteção também foi reconhecido como dificultador na adesão às $\mathrm{PP}(16)$.

Os fatores organizacionais possuem relação com a adesão às PP. A ausência e a quantidade de EPI pode ser um obstáculo para adesão às $P P^{(12,14-16,19,20)}$. Além disso, a legislação brasileira prevê a obrigatoriedade do uso de dispositivos de segurança ${ }^{(7)}$. Dessa forma, a estrutura física e a supervisão, aliadas à padronização e à socialização de rotinas, contribuem para a segurança no ambiente de trabalho(b).

Um modelo explicativo de adesão às PP que analisa uma intersecção entre fatores individuais, relativos ao trabalho e organizacionais, mostrou a associação simultânea dos mesmos $^{(24)}$. As pesquisadoras ${ }^{(24)}$ ressaltam a importância dos fatores organizacionais, com ênfase no suporte estrutural da instituição para favorecer a adoção às PP. Estudo recente, realizado em um hospital psiquiátrico(25), evidenciou que o suporte estrutural oferecido pela instituição não favoreceu a adoção às PP.

Considera-se, como limitação desta revisão, o reduzido número de produções selecionadas neste estudo. Observouse que muitos estudos ainda têm como foco a incidência 
e a caracterização dos acidentes de trabalho, com pouca ênfase sobre os fatores que têm influência para adoção de comportamentos seguros no ambiente hospitalar.

\section{CONCLUSÃO}

A presente revisão evidenciou que fatores relacionados aos próprios individuos, ao trabalho e às instituições têm associação com a adesão dos trabalhadores de saúde às PP. Esses fatores atuam tanto isoladamente como entre si, contribuindo de forma positiva ou negativa na adesão às PP. Considera-se essencial examinar esses fatores em diferentes realidades, por meio da realização de novas investigações, que poderão aprofundar a compreensão desses fenômenos.

A educação permanente dos trabalhadores foi apontada como necessária para a incorporação de práticas seguras nas instituições de saúde. Além disso, outras medidas relacionadas ao ambiente e à organização do trabalho são importantes quando se pretende alcançar uma cultura de segurança organizacional. Essas medidas incluem: melhorias na estrutura física, disposição adequada dos EPI, supervisão das práticas seguras, entre outras. Não se pode pensar em ações voltadas exclusivamente aos individuos, sem considerar o contexto e os múltiplos fatores que interferem nesses comportamentos.

A literatura analisada aponta para necessidade do compartilhamento de responsabilidades entre os trabalhadores e os serviços de saúde. Os serviços devem oferecer as condições para a execução de práticas seguras. E os trabalhadores precisam firmar o compromisso ético, reconhecendo o risco a que estão expostos e tomando as medidas preconizadas para minimizar a ocorrência de exposição ocupacional.

\section{REFERÊNCIAS}

1 - Dornelles C. Carvalho LA, Thofehrn MB, Nunes NJS, Fernandes HN. Exposição de profissionais de saúde ao material biológico: estudo no ambiente hospitalar. J Nurs Health. 2016; 1 (1): 64-75.

2 - Valim MD, Marziale MHP. Hayashida M, Richart-Martinez M. Ocorrência de acidentes de trabalho com material biológico potencialmente contaminado em enfermeiros. Acta Paul Enferm. 2014; 27 (3): 280-6.

3 - Malaguti-Toffano SE, Santos CB, Canini SRMS, et al. Adesão as precauçōespadrão de profissionais de enfermagem de um hospital universitário. Acta Paul Enferm. 2012; 25 (3): 401-7.

4 - Pereira FMV, Malaquti-Toffano SE, Silva AM, Canini SRMS, Gir E. Adesão às precauções -padrão por profissionais de enfermagem que atuam em terapia intensiva em um hospital universitário. Rev. Esc Enferm. USP. 2013; 47 (3): 686-93.

5 - Mendonça KM, Tipple AFV, Sousa ACS, Pereira MS, Rapparini C. Acidentes com material biológico em serviços de urgência e emergência. Ciencia y enfermeria. 2014: XX (2): 65-71.

6 - Neves HCC, Souza ACS, Medeiros M, Munari DB, Ribeiro LCM, Tipple AFV. Segurança dos trabalhadores de enfermagem e fatores determinantes para adesão aos equipamentos de proteção individual. Rev. Latino-Am. Enfermagem [Internet]. mar-abr 2011 [acesso em: 14 fev. 2016]; 19 (2): [08 telas]. Disponivel em: http://www. scielo.br/pdf/rlae/v19n2/pt_18

7 - Galon T, Marziale MHP. Souza WL. A legislação brasileira e as recomendações internacionais sobre a exposição ocupacional aos agentes biológicos. Rev Bras Enferm. 2011; 64 (1): 160-7.

8 - Miranda FMD, Sarquis LMM, Cruz EDA, Kirchhof ALC. Felli VEA. Crenças relacionadas aos accidentes de trabalho com fluidos biológicos. Rev Enferm UFSM. 2016; 6 (2): 235-47.

9 - Valle A, Moura MEB, Nunes BMVT. Figueiredo MLF. A Biossegurança sob o olhar de Enfermeiros. Rev. enferm. UERJ. 2012; 20 (3): 361-7.

10 - Mendes KDS, Silveira RCCP, Galvão CM. Revisão Integrativa: método de pesquisa para a incorporação de evidências na saúde e na enfermagem. Texto Contexto Enferm. 2008; 17 (4): 758-64.

11 - Frota OP, Ferreira AM, Loureiro MDR, Cheade MFM, Reis MG. O uso de equipamento de proteção individual por profissionais de enfermagem na aspiração endotraqueal. Rev. Enferm. UERJ. 2012; 10 (esp.): 625-30.

12 - Neves HCC, Souza ACS, Barbosa JM, Ribeiro LCM, Tipple, AFV, Alves, SB, et al. $\mathrm{O}$ uso de equipamentos de proteção individual por profissionais em unidades de endoscopia. Rev. Enferm UERJ. 2010; 19 (1): 61-6.
13 - Lymer U, Richt B, Isaksson B. Blood exposure: factors promoting health care workers' compliance with guidelines in connection with risk. Issues in Nursing. 2004: 13: 547-54.

14 - Osborne S. Influences on compliance with standard precautions among operating room nurses. Am J Infect Control. 2003; 31 (7): 415-23.

15 - Leliopoulou C, Waterman H, Chakrabarty S. Nurses failure to appreciate the risks of infection due to needle stick acidentes: a hospital based survey. Journal of Hospital Infection. 1999: 42: 53-9.

16 - Ribeiro LCM, Souza ACS, Neves HCC, Munari DB, Medeiros M, Tipple AFV. Influência da exposição a material biológico na adesão ao uso de equipamentos de proteção individual. Cienc Cuid Saúde. 2010; 9 (2): 325-32.

17 - Tipple AFV, Aguliari HT, Souza ACS, Pereira MS, Medonça ACC, Silveira C. Equipamentos de proteção em centros de material e esterilização: disponibilidade. uso e fatores intervenientes à adesão. Cienc Cuid Saude. 2007; 6 (4): 441-8.

18 - Knight VM, Bodsworth NJ. Perceptions and practice of universal blood and body fluid precautions by registered nurses at a major Sydney teaching hospital Journal of Advanced Nursing. 1998; 27: 746-51.

19 - Akyüz N, Keskin M, Akyolcu N, Cavdar I, Ozbas A, Ayoglu T, et al. How and how much do endoscopy professionals protect themselves agains infection? International Journal of Surgery. 2014: 12: 720-4.

20 - Efstathiou G. Papastavrou E, Raftopoulos V. Merkouris A. Compliance of Cypriot nurses with Standard Precautions to avoid exposure to pathogens. Nursing and Health Sciences. 2011; 13: 53-9.

21 - Jeong I, Cho J. Park S. Compliance with standard precautions among operating room nurses in South Korea. Am J Infect Control. 2008; 36 (10): 739-42.

22 - Madan AK, Raafat A, Hunt J, Rentz D, Wahle MJ, Flint, LM. Barrier Precautions in Trauma: Is knowledge Enough? The Journal of TRAUMA Injury. Infection and Critical Care. 2001; 52: 540-3.

23 - Lymer U, Richt B, Isaksson B. Health care workers' action strategies in situations that envolve a risk of blood exposure. Journal of Clinical Nursing. 2003; 12: 660-7.

24 - Brevidelli MM. Cianciarullo TI. Fatores psicossociais e organizacionais na adesão às precauções-padrão. Rev Saúde Pública. 2009; 43 (6): 907-16.

25 - Piai-Morais TH, Orlandi FS, Figueiredo RM. Fatores que influenciam a adesão às precauções-padrão entre profissionais de enfermagem em hospital psiquiátrico. Rev Esc Enferm USP. 2015; 49 (3): 478-85. 